\title{
Donsker type theorem for the Rosenblatt process and a binary market model
}

\author{
Soledad Torres ${ }^{1}$ Ciprian A. Tudor ${ }^{2}$ \\ ${ }^{1}$ Departamento de Estadística, Universidad de Valparaíso, \\ Casilla 123-V, 4059 Valparaiso, Chile. \\ soledad.torres@uv.cl \\ ${ }^{2}$ SAMOS/MATISSE, Centre d'Economie de La Sorbonne, \\ Université de Panthéon-Sorbonne Paris 1, \\ 90, rue de Tolbiac, 75634 Paris Cedex 13, France. \\ tudor@univ-paris1.fr
}

November 8, 2018

\begin{abstract}
In this paper, we prove a Donsker type approximation theorem for the Rosenblatt process, which is a selfsimilar stochastic process exhibiting long range dependence. By using numerical results and simulated data, we show that this approximation performs very well. We use this result to construct a binary market model driven by this process and we show that the model admits arbitrage opportunities.
\end{abstract}

2000 AMS Classification Numbers: 60F17, 91B70.

Key words: Rosenblatt process, random walk, stock price model, binary market model

\section{Introduction}

Long range dependence stochastic processes have been intensively used as models for different physical phenomena. First, these properties appeared in empirical studies in areas like hydrology and geophysics; more recently, they appeared to play an important role in network traffic analysis and telecommunications. As a consequence, efficient mathematical models based on long range dependence (or long memory) processes have been proposed in these directions.

The notions of long range dependence and selfsimilarity have also been considered in mathematical finance. An excellent survey on the different aspects of the appearance of the long range dependence in practice is the paper [3]. The debate on the presence of long 
memory in stock prices is actually not new. The idea that asset returns could exhibit long range dependence comes from Mandelbrot ([11]) and then observed in several empirical studies. We refer, among others, to [26] for concrete examples and for interesting comments on this question. We also mention that some other authors rejected the idea of the presence of long memory in asset returns (see e.g. [10]).

A rather general opinion is that long range dependence in financial models is strongly related to the presence of arbitrage. For example, in the case of market models driven by the fractional Brownian motion, this has been explicitly shown by Rogers ([17]) or Sottinen ([22]). In special situations, for example under transaction costs, arbitrage could be eliminated (see [7] or [20]). Different approaches, based on Wick-Itô calculus, have been developed in e.g. [2], [9]. Models driven by long range dependence processes others than the fractional Brownian motion have been, from the stochastic calculus point of view, less considered. This is actually one of the motivations of our work: we propose a binary market model driven by a non-Gaussian selfsimilar process (called the Rosenblatt process) which exhibits long range dependence and we show that this type of model admits arbitrage opportunities. It is actually known that in general arbitrage-free models imply the fact that the price process is a semimartingale ([5]) but it has been recently proved in [4] that by modifying the class of admissible strategies, one can also consider arbitrage-free models driven by non-martingales.

To construct our binary market model based on the Rosenblatt process we need a Donsker type theorem to approximate in law this process by some disturbed two-dimensional random walks; this results could be useful by itself. In fact, this theorem extends a result by Sottinen [22] and represents a variant of the so-called Non Central Limit Theorem proved in [6] and [24]. We mention that, since we are now in a non-Gaussian context, the proof of this result demands different techniques.

Our paper is organized as follows. In Section 2 we describe the basic properties of the Rosenblatt process. Section 3 contains the proof of the Donsker theorem to approximate weakly, in the Skorohod topology, the Rosenblatt process by walks. In Section 4, we introduce our binary market model which is showed to converge to the Black and Scholes model with Rosenblatt noise. We show that the model admits arbitrage opportunities and we construct a such opportunity.

Finally, in Appendix we present numerical results based on simulated data which show that the approximation method performs very well. Related numerical results can be found in [1] or [16].

\section{Preliminaries}

The Rosenblatt process appears as a limit in the so called Non Central Limit Theorem (see 6] or [24]). We recall the general context. Consider $\left(\xi_{n}\right)_{n \in \mathbb{Z}}$ a stationary Gaussian sequence with mean zero and covariance 1 such that its correlation function satisfies

$$
r(n):=\mathbf{E}\left(\xi_{0} \xi_{n}\right)=n^{\frac{2 H-2}{k}} L(n),
$$


with $k \geq 1$ integer, $H \in\left(\frac{1}{2}, 1\right)$ and $L$ is a slowly varying function at infinity. Denote by $H_{m}(x)$ the Hermite polynomial of degree $m$ given by $H_{m}(x)=(-1)^{m} e^{\frac{x^{2}}{2}} \frac{d^{m}}{d x^{m}} e^{-\frac{x^{2}}{2}}$. Let $g$ be a function such that $\mathbf{E}\left(g\left(\xi_{0}\right)\right)=0$ and $\mathbf{E}\left(g\left(\xi_{0}\right)^{2}\right)<\infty$. Suppose that $g$ has Hermite rank equal to $k$; that is, if $g$ admits the following expansion in Hermite polynomials

$$
g(x)=\sum_{j \geq 0} c_{j} H_{j}(x), \quad c_{j}=\frac{1}{j !} \mathbf{E}\left(g\left(\xi_{0} H_{j}\left(\xi_{0}\right)\right)\right),
$$

then

$$
k=\min \left\{j ; c_{j} \neq 0\right\} .
$$

Since $\mathbf{E}\left[g\left(\xi_{0}\right)\right]=0$, we have $k \geq 1$. The Non Central Limit Theorem ([6], 24]) says that the sequence of stochastic processes

$$
\frac{1}{n^{H}} \sum_{j=1}^{[n t]} g\left(\xi_{j}\right)
$$

converges as $n \rightarrow \infty$, in the sense of finite dimensional distributions, to the process (called the Hermite process)

$$
Z_{H}^{k}(t)=c(H, k) \int_{\mathbb{R}^{k}} \int_{0}^{t}\left(\prod_{j=1}^{k}\left(s-y_{i}\right)_{+}^{-\left(\frac{1}{2}+\frac{1-H}{k}\right)}\right) d s d B\left(y_{1}\right) \ldots d B\left(y_{k}\right),
$$

where $x_{+}=\max (x, 0)$ and the above integral is a multiple Wiener-Itô stochastic integral with respect to a Brownian motion $B(y))_{y \in \mathbb{R}}$ (see [15]).

Let us list some basic properties of the Hermite processes.

- it exhibits long-range dependence (the covariance function decay at a power function at zero -"Joseph effect")

- it is $H$-selfsimilar in the sense that for any $c>0,\left(Z_{H}^{k}(c t)\right)={ }^{(d)}\left(c^{H} Z_{H}^{k}(t)\right)$, where " $=^{(d)} "$ means equivalence of all finite dimensional distributions

- it has stationary increments, that is, the joint distribution of $\left(Z_{H}^{k}(t+h)-Z_{H}^{k}(h), t \in[0, T]\right)$ is independent of $h>0$.

- the covariance function is

$$
\mathbf{E}\left(Z_{H}^{k}(t) Z_{H}^{k}(s)\right)=\frac{1}{2}\left(t^{2 H}+s^{2 H}-|t-s|^{2 H}\right), \quad s, t \in[0, T]
$$

and consequently, for every $s, t \in[0, T]$

$$
\mathbb{E}\left|Z_{H}^{k}(t)-Z_{H}^{k}(s)\right|^{2}=|t-s|^{2 H}
$$


- the Hermite process is Holdër continuous of order $\delta<H$

- if $k \geq 2$, then $Z_{H}^{k}$ is non-Gaussian.

When $k=1$ the process given by (2) is nothing else that the fractional Brownian motion ( $f B m)$ with Hurst parameter $H \in\left(0, \frac{1}{2}\right)$. For $k \geq 2$ the process is not Gaussian. If $k=2$ then the process (2) is known as the Rosenblatt process (it has been actually named by M. Taqqu).

We focus here our attention on the case $k=2$. We will work with the representation of this processes as integral with respect to a Wiener process on a finite interval. Recall that the $\mathrm{fBm} B^{H}$ with Hurst parameter $H>\frac{1}{2}$ can be written as

$$
B_{t}^{H}=\int_{0}^{t} K^{H}(t, s) d W_{s}, \quad t \in[0, T]
$$

with $\left(W_{t}, t \in[0, T]\right)$ a standard Wiener process and

$$
K^{H}(t, s)=c_{H} s^{\frac{1}{2}-H} \int_{s}^{t}(u-s)^{H-\frac{3}{2}} u^{H-\frac{1}{2}} d u
$$

where $t>s$ and $c_{H}=\left(\frac{H(2 H-1)}{\beta\left(2-2 H, H-\frac{1}{2}\right)}\right)^{\frac{1}{2}}$. From (5) we obtain that for $t>s$,

$$
\frac{\partial K}{\partial t}(t, s)=c_{H}\left(\frac{s}{t}\right)^{\frac{1}{2}-H}(t-s)^{H-\frac{3}{2}} .
$$

Aa analogous representation for the Rosenblatt process has been given in [25]. We have

$$
Z_{H}^{2}(t):=Z_{t}=^{(d)} d(H) \int_{0}^{t} \int_{0}^{t}\left[\int_{y_{1} \vee y_{2}}^{t} \frac{\partial K^{H^{\prime}}}{\partial u}\left(u, y_{1}\right) \frac{\partial K^{H^{\prime}}}{\partial u}\left(u, y_{2}\right) d u\right] d W_{y_{1}} d W_{y_{2}}
$$

where $\left(W_{t}, t \in[0, T]\right)$ is a Brownian motion,

$$
H^{\prime}=\frac{H+1}{2}
$$

and $d(H)=\frac{1}{H+1}\left(\frac{H}{2(2 H-1)}\right)^{-\frac{1}{2}}$. Note that $H>\frac{1}{2}$ implies $H^{\prime}>\frac{3}{4}$.

\section{Convergence in law to the Rosenblatt process}

This part in consecrated to a Donsker invariance principle for the Rosenblatt process. From now on, we will consider the Rosenblatt process to be given by the formula (7). We will denote, for every $t \in[0, T]$

$$
F\left(t, y_{1}, y_{2}\right)=d(H) 1_{[0, t]}\left(y_{1}\right) 1_{[0, t]}\left(y_{2}\right) \int_{y_{1} \vee y_{2}}^{t} \frac{\partial K^{H^{\prime}}}{\partial u}\left(u, y_{1}\right) \frac{\partial K^{H^{\prime}}}{\partial u}\left(u, y_{2}\right) d u
$$


and then

$$
Z_{t}=\int_{0}^{T} \int_{0}^{T} F\left(t, y_{1}, y_{2}\right) d W\left(y_{1}\right) d W\left(y_{2}\right), \quad t \in[0, T] .
$$

The kernel $K^{H^{\prime}}$ (denoted simply by $K$ in the sequel) is the standard kernel of the fractional Brownian motion (5).

Let us first recall some known facts. Consider $\left(\xi_{i}\right)_{i \geq 1}$ a sequence of i.i.d random variables with $\mathbb{E}\left(\xi_{i}\right)=0$ and $\mathbb{E}\left(\xi_{i}^{2}\right)=1$. The Donsker Invariance Principle says that the sequence of processes

$$
W_{t}^{n}=\frac{1}{\sqrt{n}} \sum_{i=1}^{[n t]} \xi_{i}
$$

converges weakly, in the Skorohod topology, to a standard Brownian motion. Here $[x]$ denotes the biggest integer smaller than $x$.

This result has been extended in [22] to the fractional Brownian motion (see also [12]). Define

$$
K^{n}(t, s):=n \int_{s-\frac{1}{n}}^{s} K\left(\frac{[n t]}{n}, u\right) d u, \quad n \geq 1
$$

and put

$$
B_{t}^{n}=\int_{0}^{t} K^{n}(t, s) d W_{s}^{n}=\sum_{i=1}^{[n t]} n \int_{\frac{i-1}{n}}^{\frac{i}{n}} K\left(\frac{[n t]}{n}, s\right) d s \frac{\xi_{i}}{\sqrt{n}}, \quad n \geq 1 .
$$

Then it has been proved in [22] that the disturbed random walk $B^{n}$ converges weakly to the fractional Brownian motion.

From the above results and the representation (9) it is quite natural to define the following approximation for the Rosenblatt process

$$
Z_{t}^{n}=\sum_{i, j=1 ; i \neq j}^{[n t]} n^{2} \int_{\frac{i-1}{n}}^{\frac{i}{n}} \int_{\frac{j-1}{n}}^{\frac{j}{n}} F\left(\frac{[n t]}{n}, u, v\right) d v d u \frac{\xi_{i}}{\sqrt{n}} \frac{\xi_{j}}{\sqrt{n}}, \quad t \in[0, T] .
$$

Remark 1 We eliminate the diagonal " $i=j$ " because the Rosenblatt process is defined as a double Wiener-Itô integral and as a consequence it has zero mean. When the diagonal $i=j$ is included in the sum (11) then the limit is in general a double Stratonovich integral (see [8] or [21]).

Proposition 1 The family of stochastic processes $\left(Z_{t}^{n}\right)_{t \in[0, T]}$ converges in the sense of finite dimensional distributions to the process $\left(Z_{t}\right)_{t \in[0, T]}$ (9)).

Proof: We will proof this result in several steps. 
Step 1: Let us consider an arbitrary sequence of partitions of the interval $[0, T]$ of the form

$$
\pi^{m}: 0=t_{0}^{m}<t_{1}^{m}<\ldots<t_{m}^{m}=T
$$

with $\left|\pi^{m}\right| \rightarrow 0$ as $m \rightarrow \infty$. Define

$$
Z_{t}^{\pi^{m}}=\sum_{i, j=1 ; i \neq j}^{m} \frac{1}{\left|\Delta_{i}^{m}\right|\left|\Delta_{j}^{m}\right|}\left(\int_{\Delta_{i}^{m}} \int_{\Delta_{j}^{m}} F(t, u, v) d v d u\right) W\left(\Delta_{i}^{m}\right) W\left(\Delta_{j}^{m}\right)
$$

where we denoted by $\Delta_{i}^{m}=\left[t_{i-1}^{m}, t_{i}^{m}\right)$ and by

$$
W\left(\Delta_{i}^{m}\right)=W_{t_{i}^{m}}-W_{t_{i-1}^{m}}
$$

Then it follows from [21, Theorem 3.4, or [8] that for fixed $t$ the sequence $Z_{t}^{\pi^{m}}$ converges in $L^{2}(\Omega)$ as $\left|\pi^{m}\right| \rightarrow 0$ to the multiple Wiener-Itô integral of $F(t, \cdot)$ with respect to the Brownian motion $W$

$$
\int_{0}^{T} \int_{0}^{T} F(t, u, v) d W_{u} d W_{v}=Z_{t}
$$

Step 2: Secondly, define the process

$$
Z_{t}^{\pi^{m}, n}=\sum_{i, j=1 ; i \neq j}^{m} \frac{1}{\left|\Delta_{i}^{m}\right|\left|\Delta_{j}^{m}\right|}\left(\int_{\Delta_{i}^{m}} \int_{\Delta_{j}^{m}} F(t, u, v) d v d u\right) W^{n}\left(\Delta_{i}^{m}\right) W^{n}\left(\Delta_{j}^{m}\right)
$$

where $W^{n}$ is the random walk given by (10). Then clearly, for fixed $m$, as $n$ goes to $\infty$, the finite dimensional distributions of $Z^{\pi^{m}, n}$ converges to the finite dimensional distributions of $Z^{\pi^{m}}$ (this comes from the weak convergence of $W^{n}$ to the Wiener process $W$ ).

Step 3: We prove now that for every $t \in[0, T]$, the sequence $Z_{t}^{\pi^{m}, n}$ converges in $L^{2}(\Omega)$ to $Z_{t}^{\prime}, n$ as $m \rightarrow \infty$, where

$$
Z_{t}^{\prime}, n=\sum_{i, j=1 ; i \neq j}^{[n t]} n^{2} \int_{\frac{i-1}{n}}^{\frac{i}{n}} \int_{\frac{j-1}{n}}^{\frac{j}{n}} F(t, u, v) d v d u \frac{\xi_{i}}{\sqrt{n}} \frac{\xi_{j}}{\sqrt{n}}, \quad t \in[0, T] .
$$

Consider the sequence

$$
F^{\pi^{m}}(t, u, v)=\sum_{i, j=1 ; i \neq j}^{m} \frac{1}{\left|\Delta_{i}^{m}\right|\left|\Delta_{j}^{m}\right|}\left(\int_{\Delta_{i}^{m}} \int_{\Delta_{j}^{m}} F(t, u, v) d v d u\right) 1_{\Delta_{i}^{m}}(u) 1_{\Delta_{j}^{m}}(v) .
$$

Then $F^{\pi^{m}}(t, \cdot)$ converges to $F(t, \cdot)$ in $L^{2}\left([0, T]^{2}\right)$ as $m \rightarrow \infty$ (see [21], [8]).

First note that $Z_{t}^{\prime}, n$ can be approximated in $L^{2}(\Omega)$ as $m \rightarrow \infty$ by

$$
Z_{t}^{\prime}, \pi^{m}, n=\sum_{k, l=1 ; k \neq l}^{[n t]} n^{2} \int_{\frac{k-1}{n}}^{\frac{k}{n}} \int_{\frac{l-1}{n}}^{\frac{l}{n}} F^{\pi^{m}}(t, u, v) d v d u \frac{\xi_{k}}{\sqrt{n}} \frac{\xi_{l}}{\sqrt{n}}
$$


Indeed,

$$
\begin{aligned}
& \mathbb{E}\left|Z_{t}^{\prime} \pi^{m}, n-Z_{t}^{\prime}, n\right|^{2} \\
= & \sum_{k, l=1 ; k \neq l}^{[n t]} n^{2}\left(\int_{\frac{k-1}{n}}^{\frac{k}{n}} \int_{\frac{l-1}{n}}^{\frac{l}{n}}\left(F^{\pi^{m}}(t, u, v)-F(t, u, v)\right) d v d u\right)^{2} \mathbb{E}\left(\xi_{k}^{2}\right) \mathbb{E}\left(\xi_{l}^{2}\right) \\
\leq & \sum_{k, l=1 ; k \neq l}^{[n t]} \int_{\frac{k-1}{n}}^{\frac{k}{n}} \int_{\frac{l-1}{n}}^{\frac{l}{n}}\left(F^{\pi^{m}}(t, u, v)-F(t, u, v)\right)^{2} d v d u \\
\leq & \int_{0}^{T} \int_{0}^{T}\left(F^{\pi^{m}}(t, u, v)-F(t, u, v)\right)^{2} d v d u
\end{aligned}
$$

and this clearly goes to zero as $m \rightarrow \infty$.

It remains to observe that $Z_{t}^{\prime}, \pi^{m}, n$ is equal to $Z_{t}^{\pi^{m}}$ for every $t, m, n$. We can write, if $\lambda$ denotes the Lebesque measure,

$$
\begin{aligned}
& Z_{t}^{\prime}, \pi^{m}, n=\sum_{k, l=1 ; k \neq l}^{[n t]} n^{2} \int_{\frac{k-1}{n}}^{\frac{k}{n}} \int_{\frac{l-1}{n}}^{\frac{l}{n}} d v d u \\
& \times\left(\sum_{i, j=1 ; i \neq j}^{m} \frac{1}{\left|\Delta_{i}^{m}\right|\left|\Delta_{j}^{m}\right|}\left(\int_{\Delta_{i}^{m}} \int_{\Delta_{j}^{m}} F(t, x, y) d y d x\right) 1_{\Delta_{i}^{m}}(u) 1_{\Delta_{j}^{m}}(v)\right) \frac{\xi_{k}}{\sqrt{n}} \frac{\xi_{l}}{\sqrt{n}} \\
& =\sum_{i, j=1 ; i \neq j}^{m} \frac{1}{\left|\Delta_{i}^{m}\right|\left|\Delta_{j}^{m}\right|}\left(\int_{\Delta_{i}^{m}} \int_{\Delta_{j}^{m}} F(t, x, y) d y d x\right) \\
& \times \sum_{k, l=1 ; k \neq l}^{[n t]} n^{2} \frac{\xi_{k}}{\sqrt{n}} \frac{\xi_{l}}{\sqrt{n}} \int_{\frac{k-1}{n}}^{\frac{k}{n}} \int_{\frac{l-1}{n}}^{\frac{l}{n}} 1_{\Delta_{i}^{m}}(u) 1_{\Delta_{j}^{m}}(v) d v d u \\
& =\sum_{i, j=1 ; i \neq j}^{m} \frac{1}{\left|\Delta_{i}^{m}\right|\left|\Delta_{j}^{m}\right|}\left(\int_{\Delta_{i}^{m}} \int_{\Delta_{j}^{m}} F(t, x, y) d y d x\right) \\
& \times \sum_{k, l=1 ; k \neq l}^{[n t]} n^{2} \frac{\xi_{k}}{\sqrt{n}} \frac{\xi_{l}}{\sqrt{n}} \lambda\left(\left[\frac{k-1}{n}, \frac{k}{n}\right) \bigcap \Delta_{i}^{m}\right) \lambda\left(\left[\frac{l-1}{n}, \frac{l}{n}\right) \bigcap \Delta_{j}^{m}\right) \\
& =\sum_{i, j=1 ; i \neq j}^{m} \frac{1}{\left|\Delta_{i}^{m}\right|\left|\Delta_{j}^{m}\right|}\left(\int_{\Delta_{i}^{m}} \int_{\Delta_{j}^{m}} F(t, x, y) d y d x\right) \\
& \times \sum_{k ;\left[\frac{k-1}{n}, \frac{k}{n}\right) \subset \Delta_{i}^{m}} \sum_{l \neq k ;\left[\frac{l-1}{n}, \frac{l}{n}\right) \subset \Delta_{j}^{m}} \frac{\xi_{k}}{\sqrt{n}} \frac{\xi_{l}}{\sqrt{n}}
\end{aligned}
$$


and on the other hand by using (12) and (10), one has

$$
Z_{t}^{\pi^{m}, n}=\sum_{i, j=1 ; i \neq j}^{m} \frac{1}{\left|\Delta_{i}^{m}\right|\left|\Delta_{j}^{m}\right|}\left(\int_{\Delta_{i}^{m}} \int_{\Delta_{j}^{m}} F(t, x, y) d y d x\right) \sum_{k=\left[n t_{i-1}^{m}\right]+1}^{\left[n t_{i}^{m}\right]} \sum_{l=\left[n t_{j-1}^{m}\right]+1}^{\left[n t_{j}^{m}\right]} \frac{\xi_{k}}{\sqrt{n}} \frac{\xi_{l}}{\sqrt{n}}
$$

and it is not difficult to see that $Z_{t}^{\pi^{m}, n}$ and $Z_{t}^{\prime}, \pi^{m}, n$ coincide.

Step 4: At this point we conclude that the family of processes $Z^{\prime}, n$ converges in the sense of finite dimensional distributions to the Rosenblatt process $Z_{t}$. Let $h$ be a function defined on $\mathbb{R}^{p}$ and consider $s_{1}, \ldots, s_{p} \in[0, T]$. We will show that

$$
\mathbb{E}\left(h\left(Z_{s_{1}}^{\prime}, \ldots, Z_{s_{p}}^{\prime}, n\right)\right)-\mathbb{E}\left(h\left(Z_{s_{1}}, \ldots, Z_{s_{p}}\right)\right)
$$

converges to zero as $n \rightarrow \infty$. This can be bounded by $A+B+C$ where

$$
\begin{gathered}
A=\left|\mathbb{E}\left(h\left(Z_{s_{1}}, \ldots, Z_{s_{p}}\right)\right)-\mathbb{E}\left(h\left(Z_{s_{1}}^{\pi^{m}}, \ldots, Z_{s_{p}}^{\pi^{m}}\right)\right)\right| \\
B=\left|\mathbb{E}\left(h\left(Z_{s_{1}}^{\pi^{m}, n}, \ldots, Z_{s_{p}}^{\pi^{m}, n}\right)\right)-\mathbb{E}\left(h\left(Z_{s_{1}}^{\pi^{m}}, \ldots, Z_{s_{p}}^{\pi^{m}}\right)\right)\right|
\end{gathered}
$$

and

$$
C=\left|\mathbb{E}\left(h\left(Z_{s_{1}}^{\pi^{m}, n}, \ldots, Z_{s_{p}}^{\pi^{m}, n}\right)\right)-\mathbb{E}\left(h\left(Z_{s_{1}^{\prime}, n}^{\prime}, \ldots, Z_{s_{p}}^{\prime, n}\right)\right)\right| .
$$

By Step 1 and Step 3 we have that for fixed $c>0$ the terms $A$ and $C$ are bounded (uniformly in $n$ ) by $c$ when $m$ is large enough. the term $B$ tends to zero as $n \rightarrow \infty$ from Step 2 .

Step 5: Clearly the family $Z^{\prime}, n$ can be replaced by the family $Z^{n}$ (11) because their difference goes to zero in $L^{2}(\Omega)$ as $n \rightarrow \infty$.

Next, we show the tightness.

Proposition 2 The family $Z^{n}$ given by (11) is tight.

Proof: Let $s<t, s, t \in[0, T]$. It holds, since the kernel $F(s, u, v)$ vanishes when $u$ or $v$ are 
bigger that $s$,

$$
\begin{aligned}
& \mathbb{E}\left|Z_{t}^{n}-Z_{s}^{n}\right|^{2} \\
= & \mathbb{E}\left(\sum_{i, j=1 ; i \neq j}^{[n t]} n^{2} \int_{\frac{i-1}{n}}^{\frac{i}{n}} \int_{\frac{j-1}{n}}^{\frac{j}{n}}\left[F\left(\frac{[n t]}{n}, u, v\right)-F\left(\frac{[n s]}{n}, u, v\right)\right] d v d u \frac{\xi_{i}}{\sqrt{n}} \frac{\xi_{j}}{\sqrt{n}}\right)^{2} \\
= & \sum_{i, j=1 ; i \neq j}^{[n t]} n^{2}\left(\int_{\frac{i-1}{n}}^{\frac{i}{n}} \int_{\frac{j-1}{n}}^{\frac{j}{n}}\left[F\left(\frac{[n t]}{n}, u, v\right)-F\left(\frac{[n s]}{n}, u, v\right)\right] d v d u\right)^{2} \\
\leq & \sum_{i, j=1 ; i \neq j}^{[n t]} \int_{\frac{i-1}{n}}^{\frac{i}{n}} \int_{\frac{j-1}{n}}^{\frac{j}{n}}\left[F\left(\frac{[n t]}{n}, u, v\right)-F\left(\frac{[n s]}{n}, u, v\right)\right]^{2} d v d u \\
\leq & \int_{0}^{T} \int_{0}^{T}\left[F\left(\frac{[n t]}{n}, u, v\right)-F\left(\frac{[n s]}{n}, u, v\right)\right]^{2} d v d u \\
= & \left|\frac{[n t]}{n}-\frac{[n s]}{n}\right|^{2} .
\end{aligned}
$$

Now the conclusion follows by using exactly the same arguments as in 22, end of the proof of Theorem 1 .

The main result of this section is a consequence of Proposition 1 and Proposition 2 ,

Theorem 1 The family of stochastic processes (11) converges weakly, in the Skorohod topology, to the Rosenblatt process.

\section{Binary market model based on the Rosenblatt process}

The binary market constitutes a financial model where the asset are traded at discrete times. In classical cases (for example when the driven process is the Wiener process) the binary model approximates the Black and Scholes model.

Let us start by introducing the Black and Scholes model driven by the Rosenblatt process. As usually, we will consider two assets: a safe investment satisfying

$$
B_{t}=\int_{0}^{t} r_{s} B_{s} d s
$$

where $r$ is a differentiable deterministic function and a risky asset with price dynamic following the stochastic equation

$$
S_{t}=S_{0}+\int_{0}^{t} a_{s} S_{s} d s+\sigma \int_{0}^{t} S_{s} d Z_{s}
$$

where $Z$ is a Rosenblatt process, $\sigma>0$ and $a$ is a differentiable deterministic function. The integral with respect to $Z$ is understood here in a pathwise sense. Since the trajectories of 
the Rosenblatt process are enough regular (in fact, they are Hölder continuous of order $\delta<H$ and $H>\frac{1}{2}$ ) it is possible to consider pathwise integrals with respect to it and to solve some stochastic equations in the pathwise sense. We refer, among others, to [13], 27] or [18]. In particular, the solution of (16) is given by (see [14], 27], [13]

$$
S_{t}=S_{0} e^{\int_{0}^{t} a_{s} d s+\sigma Z_{t}}, \quad t \in[0, T]
$$

Clearly the solution of (15) is

$$
B_{t}=B_{0} e^{\int_{0}^{t} r_{s} d s}, \quad t \in[0, T]
$$

Moreover, we will assume in the sequel that the interest rates $a$ and $r$ are deterministic bounded functions.

Let us describe now the binary market model with Rosenblatt influence. The two assets are traded now at successive times periods $t_{1}<t_{2}<\ldots<\ldots$ and their dynamics are given by

$$
B_{n}=\left(1+r_{n}\right) B_{n-1}
$$

and

$$
S_{n}=\left(a_{n}+\left(1+X_{n}\right)\right) S_{n-1}
$$

That means that $B_{n}$ and $S_{n}$ represent the prices of the bond and of the stock in the period between $t_{n}$ and $t_{n+1}$ and $r_{n}$ and $a_{n}$ are the interest rates valuable in this period. The stochastic process $X$ is binary, that is, given $X_{n-1}$ it can takes at time $n$ two possible values denoted by $d_{n}$ ("down") and $u_{n}$ ("up"). The binary market excludes arbitrage opportunities if for every $n$ it holds that (see [22])

$$
d_{n}<r_{n}-a_{n}<u_{n}
$$

In the following we will choose our binary model to be determined by

$$
X_{n}=\Delta Z_{\frac{n}{N}}^{N}
$$

where $Z^{N}$ is defined by (11) and

$$
r_{n}=\frac{1}{N} r_{\frac{n}{N}} \text { and } a_{n}=\frac{1}{N} a_{\frac{n}{N}}
$$

where $a$ and $r$ are the interest rates appearing in (15) and (16).

We have

Proposition 3 The binary market model with $X$ a and $r$ given by (20) and (21) converges as $N \rightarrow \infty$ to the Black and Scholes model given by (15) and (16)). 
Proof: Let us consider the jump

$$
\Delta Z_{t}^{N}=Z_{t}^{N}-Z_{t-}^{N}
$$

and the quadratic variation

$$
\left[Z^{N}\right]_{t}=\sum_{s \leq t}\left(\Delta Z_{s}^{N}\right)^{2}
$$

We will show that the process $\left[Z^{N}\right]$ converges in $L^{1}([0, T] \times \Omega)$ to zero. Then the conclusion will follow exactly as in [22], proof of Lemma 1.

We have, since the jumps are at times $\frac{k}{N}, k$ integer,

$$
\mathbb{E}\left|\Delta Z_{t}^{N}\right|^{2} \leq \mathbb{E}\left|Z_{t}-Z_{t-\frac{1}{N}}\right|^{2} \leq \frac{1}{N^{2 H}}
$$

and then

$$
\mathbb{E}\left[Z^{N}\right]_{t} \leq N t \frac{1}{N^{2 H}}=t N^{1-2 H}
$$

This implies that

$$
\int_{0}^{T}\left[Z^{N}\right]_{s} d s \leq c(T) N^{1-2 H}
$$

which goes to 0 as $N$ goes to $\infty$.

The next step is to show that the market admits arbitrage opportunities. Clearly, we have

$$
X_{n}=\sigma N \sum_{i, j=1 ; i \neq j}^{n}\left(\int_{\frac{i-1}{N}}^{\frac{i}{N}} \int_{\frac{j-1}{N}}^{\frac{j}{N}}\left(F\left(\frac{n}{N}, u, v\right)-F\left(\frac{n-1}{N}, u, v\right)\right) d v d u\right) \xi_{i} \xi_{j}
$$

and we will take the random variables $\xi$ to be binary, that is

$$
P(\xi=1)=P\left(\xi_{i}=-1\right)=\frac{1}{2}
$$

for every $i \geq 1$. We can write, by isolating the part involving $\xi_{n}$,

$$
X_{n}=f_{n-1}\left(\xi_{1}, \ldots, \xi_{n-1}\right)+\xi_{n} g_{n-1}\left(\xi_{1}, \ldots, \xi_{n-1}\right)
$$

where for every $n \geq 2$

$$
f_{n-1}\left(x_{1}, \ldots, x_{n-1}\right)=\sigma N \sum_{i, j=1 ; i \neq j}^{n-1}\left(\int_{\frac{i-1}{N}}^{\frac{i}{N}} \int_{\frac{j-1}{N}}^{\frac{j}{N}}\left(F\left(\frac{n}{N}, u, v\right)-F\left(\frac{n-1}{N}, u, v\right)\right) d v d u\right) x_{i} x_{j}
$$


and

$$
g_{n-1}\left(x_{1}, \ldots, x_{n-1}\right)=2 \sigma N \sum_{i=1}^{n-1}\left(\int_{\frac{i-1}{N}}^{\frac{i}{N}} \int_{\frac{n-1}{N}}^{\frac{n}{N}} F\left(\frac{n}{N}, u, v\right) d v d u\right) x_{i} .
$$

Then obviously

$$
u_{n}=f_{n-1}\left(\xi_{1}, \ldots, \xi_{n-1}\right)+g_{n-1}\left(\xi_{1}, \ldots, \xi_{n-1}\right)
$$

and

$$
d_{n}=f_{n-1}\left(\xi_{1}, \ldots, \xi_{n-1}\right)-g_{n-1}\left(\xi_{1}, \ldots, \xi_{n-1}\right) .
$$

The last result of our paper is the following.

Proposition 4 The binary market model with (21) and (20) admits arbitrage.

Proof: Throughout this proof, we will denote by $c(H)$ a generic constant depending only on $H$. Let us show now that the condition (19) fails for some $n \geq 2$. We will actually prove that the sequence

$$
f_{n-1}(1,1, \ldots, 1)-g_{n-1}(1,1, \ldots 1) \rightarrow_{n \rightarrow \infty} \infty
$$

and then clearly (19) does not hold because $r_{n}$ and $a_{n}$ are assumed to be bounded.

We have

$$
\begin{aligned}
f_{n-1}(1,1, \ldots, 1)= & \sigma N \sum_{i, j=1 ; i \neq j}^{n-1}\left(\int_{\frac{i-1}{N}}^{\frac{i}{N}} \int_{\frac{j-1}{N}}^{\frac{j}{N}}\left(F\left(\frac{n}{N}, u, v\right)-F\left(\frac{n-1}{N}, u, v\right)\right) d v d u\right) \\
= & \sigma N \sum_{i, j=1 ; i}^{n-1}\left(\int_{\frac{i-1}{N}}^{\frac{i}{N}} \int_{\frac{j-1}{N}}^{\frac{j}{N}}\left(F\left(\frac{n}{N}, u, v\right)-F\left(\frac{n-1}{N}, u, v\right)\right) d v d u\right) \\
& -\sum_{i=1}^{n-1}\left(\int_{\frac{i-1}{N}}^{\frac{i}{N}} \int_{\frac{i-1}{N}}^{\frac{i}{N}}\left(F\left(\frac{n}{N}, u, v\right)-F\left(\frac{n-1}{N}, u, v\right)\right) d v d u\right) \\
:= & \sigma N(A-B) .
\end{aligned}
$$


Using the expression of the kernel $F$ and ([6), the term $A$ can be minorized as follows

$$
\begin{aligned}
A & =c(H) \int_{0}^{\frac{n-1}{N}} \int_{0}^{\frac{n-1}{N}} \int_{\frac{n-1}{N}}^{\frac{n}{N}}(a-u)^{H^{\prime}-\frac{3}{2}}(a-v)^{H^{\prime}-\frac{3}{2}} a^{2 H^{\prime}-1} u^{\frac{1}{2}-H^{\prime}} v^{\frac{1}{2}-H^{\prime}} d a d v d u \\
& \geq c(H)\left(\frac{n-1}{N}\right)^{1-2 H^{\prime}} \int_{0}^{\frac{n-1}{N}} \int_{0}^{\frac{n-1}{N}} \int_{\frac{n-1}{N}}^{\frac{n}{N}}(a-u)^{H^{\prime}-\frac{3}{2}}(a-v)^{H^{\prime}-\frac{3}{2}} a^{2 H^{\prime}-1} d a d v d u \\
& \geq c(H)\left(\frac{n-1}{N}\right)^{1-2 H^{\prime}} \int_{\frac{n-1}{N}}^{\frac{n}{N}} d a\left(a^{H^{\prime}-\frac{1}{2}}-\left(a-\frac{n-1}{N}\right)^{H^{\prime}-\frac{1}{2}}\right)^{2} a^{2 H^{\prime}-1} \\
& \geq c(H)\left(\frac{n-1}{N}\right)^{1-2 H^{\prime}} \int_{\frac{n-1}{N}}^{\frac{n}{N}} d a\left(\frac{(n-1)^{H^{\prime}-\frac{1}{2}}-1}{N^{H^{\prime}-\frac{1}{2}}}\right)^{2} a^{2 H^{\prime}-1} \\
& \geq c(H) \frac{1}{N^{2 H^{\prime}}}(n-1)^{1-2 H^{\prime}}\left((n-1)^{H^{\prime}-\frac{1}{2}}-1\right)^{2}\left(n^{2 H^{\prime}}-(n-1)^{2 H^{\prime}}\right) \\
& =c(H) \frac{1}{N^{2 H^{\prime}}} o\left(n^{2 H^{\prime}-1}\right) .
\end{aligned}
$$

We majorize now the term $B$. We can write

$$
\begin{aligned}
B & \leq c(H) \sum_{i=1}^{n-1} \int_{\frac{i-1}{N}}^{\frac{i}{N}} \int_{\frac{i-1}{N}}^{\frac{i}{N}} \int_{\frac{n-1}{N}}^{\frac{n}{N}}(a-u)^{H^{\prime}-\frac{3}{2}}(a-v)^{H^{\prime}-\frac{3}{2}} a^{2 H^{\prime}-1} u^{\frac{1}{2}-H^{\prime}} v^{\frac{1}{2}-H^{\prime}} d a d v d u \\
& \leq c(H)\left(\frac{n}{N}\right)^{2 H^{\prime}-1} \sum_{i=1}^{n-1} \int_{\frac{i-1}{N}}^{\frac{i}{N}} \int_{\frac{i-1}{N}}^{\frac{i}{N}} \int_{\frac{n-1}{N}}^{\frac{n}{N}}\left(a-\frac{i}{N}\right)^{2 H^{\prime}-3} u^{\frac{1}{2}-H^{\prime}} v^{\frac{1}{2}-H^{\prime}} d a d v d u \\
& \leq c(H)\left(\frac{n}{N}\right)^{2 H^{\prime}-1} \sum_{i=1}^{n-1} \int_{\frac{i-1}{N}}^{\frac{i}{N}} \int_{\frac{i-1}{N}}^{\frac{i}{N}} u^{\frac{1}{2}-H^{\prime}} v^{\frac{1}{2}-H^{\prime}} \frac{(n-i)^{2 H^{\prime}-2}-(n-(i-1))^{2 H^{\prime}-2}}{N^{2 H^{\prime}-2}} d v d u \\
& \leq c(H)\left(\frac{n}{N}\right)^{2 H^{\prime}-1} \sum_{i=1}^{n-1}\left(\left(\frac{i}{N}\right)^{\frac{3}{2}-H^{\prime}}-\left(\frac{i-1}{N}\right)^{\frac{3}{2}-H^{\prime}}\right)^{2} \frac{(n-i)^{2 H^{\prime}-2}-(n-(i-1))^{2 H^{\prime}-2}}{N^{2 H^{\prime}-2}} \\
& \leq c(H)\left(\frac{n}{N}\right)^{2 H^{\prime}-1} \frac{1}{N^{3-2 H^{\prime}}}\left(\frac{n-1}{N}\right)^{2 H^{\prime}-2} \\
& \leq c(H) \frac{1}{N^{2 H^{\prime}}} o\left(n^{4 H^{\prime}-3}\right) .
\end{aligned}
$$

From the above computations we obtain that $f_{n-1}(1, \ldots, 1)$ converges to $\infty$ as $n \rightarrow \infty$ because $2 H^{\prime}-1>4 H^{\prime}-3$. Let us estimate the term $g_{n-1}(1, \ldots, 1)$. 


$$
\begin{aligned}
& g_{n-1}(1, \ldots, 1) \\
\leq & 2 \sigma N \sum_{i=1}^{n-1}\left(\int_{\frac{i-1}{N}}^{\frac{i}{N}} \int_{\frac{n-1}{N}}^{\frac{n}{N}} F\left(\frac{n}{N}, u, v\right) d v d u\right) \\
\leq & 2 \sigma N c(H)\left(\frac{n}{N}\right)^{2 H^{\prime}-1} \sum_{i=1}^{n-1} \int_{\frac{i-1}{N}}^{\frac{i}{N}} \int_{\frac{n-1}{N}}^{\frac{n}{N}} \int_{v}^{\frac{n}{N}} u^{\frac{1}{2}-H^{\prime}} v^{\frac{1}{2}-H^{\prime}}\left(\frac{n-1}{N}-u\right)^{H^{\prime}-\frac{3}{2}}(a-v)^{H^{\prime}-\frac{3}{2}} d a d v d u \\
\leq & 2 \sigma N c(H)\left(\frac{n}{N}\right)^{2 H^{\prime}-1}\left(\frac{n-1}{N}\right)^{\frac{1}{2}-H^{\prime}} \sum_{i=1}^{n-1} \int_{\frac{i-1}{N}}^{\frac{i}{N}} \int_{\frac{n-1}{N}}^{\frac{n}{N}} u^{\frac{1}{2}-H^{\prime}}\left(\frac{n-1}{N}-u\right)^{H^{\prime}-\frac{3}{2}}\left(\frac{n}{N}-v\right)^{H^{\prime}-\frac{1}{2}} d v d u \\
\leq & 2 \sigma N c(H)\left(\frac{n}{N}\right)^{2 H^{\prime}-1}\left(\frac{n-1}{N}\right)^{\frac{1}{2}-H^{\prime}} \int_{0}^{\frac{n-1}{N}} u^{\frac{1}{2}-H^{\prime}}\left(\frac{n-1}{N}-u\right)^{H^{\prime}-\frac{3}{2}} d u \\
\leq & 2 \sigma N c(H) \frac{1}{N^{2 H^{\prime}-1}} o\left(n^{H^{\prime}-\frac{1}{2}}\right)
\end{aligned}
$$

because the integral $\int_{0}^{\frac{n-1}{N}} u^{\frac{1}{2}-H^{\prime}}\left(\frac{n-1}{N}-u\right)^{H^{\prime}-\frac{3}{2}} d u$ is equal to $\beta\left(\frac{3}{2}-H^{\prime}, H^{\prime}-\frac{1}{2}\right)$. We then obtain (24).

Comments: i) A concrete arbitrage opportunity can be easily described. For example, suppose that $a>r$. Ar a certain time $n_{0}$ we have $d_{n_{0}}>0$ because of (24); suppose that the stock price was increasing up to this time $n_{0}$. Then, buy $M$ stocks and your wealth at time $n_{0}+1$ will be positive since $M S_{n_{0}+1}>M S_{n_{0}}$.

ii) We cannot expect to have no-arbitrage when $H \in \frac{1}{2}$ as in the fractional Brownian motion case because now the limit process at $H=\frac{1}{2}$ is not necessarily a martingale. 


\section{Appendix: Monte Carlo Simulation}

This simulation study is intended to show our proposed model using simulated data. We simulate data using different values for $H$. Below, we describe the procedure used in generating the data to be used in the simulation study.

We have implemented this simulation on a standard personal computing platform (PC), and have observed that it performs very well using simulated data as can be seen from the simulated data in the figures 1 and 2 below. Despite the apparent algebraic complexity of the equations (11), the problem poses no difficulty for standard symbolic algebra packages. Using Matlab's simulations and algebra capabilities yielded the best computing times. In our implementation, which performs an iteration of the algorithm from $i=0$ to $i=n$. Figure 1 shows the histogram for a fixed time $t$ for the marginal density of the Rosenblatt process. We can see the skew structure of the distribution.
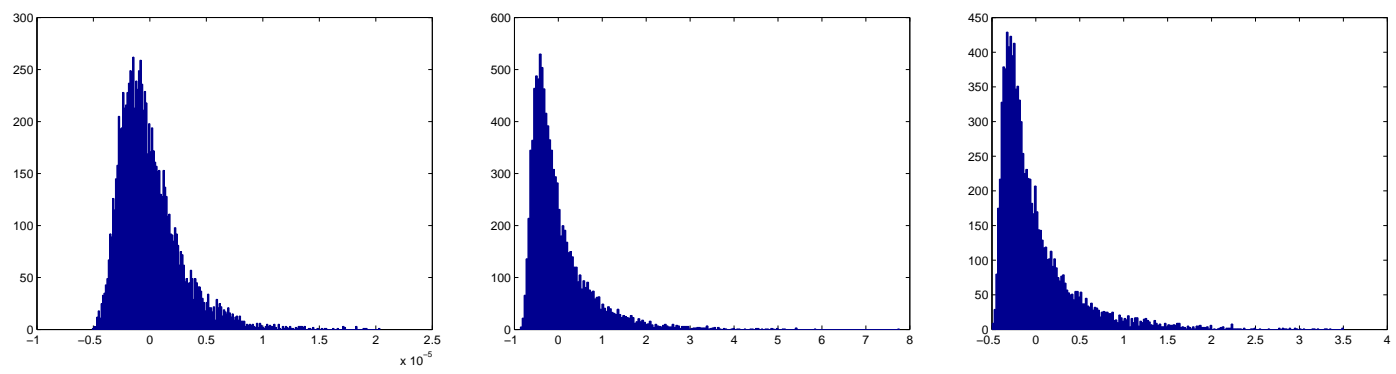

Figure 1: Marginal distributions for $H=0.5 ; H=0.8$ and $H=0.9$.

Figure 2 shows the some paths of the discretization for the Rosenblatt process. We use the values for the parameter $H(H=0.8$ and $H=0.9)$.
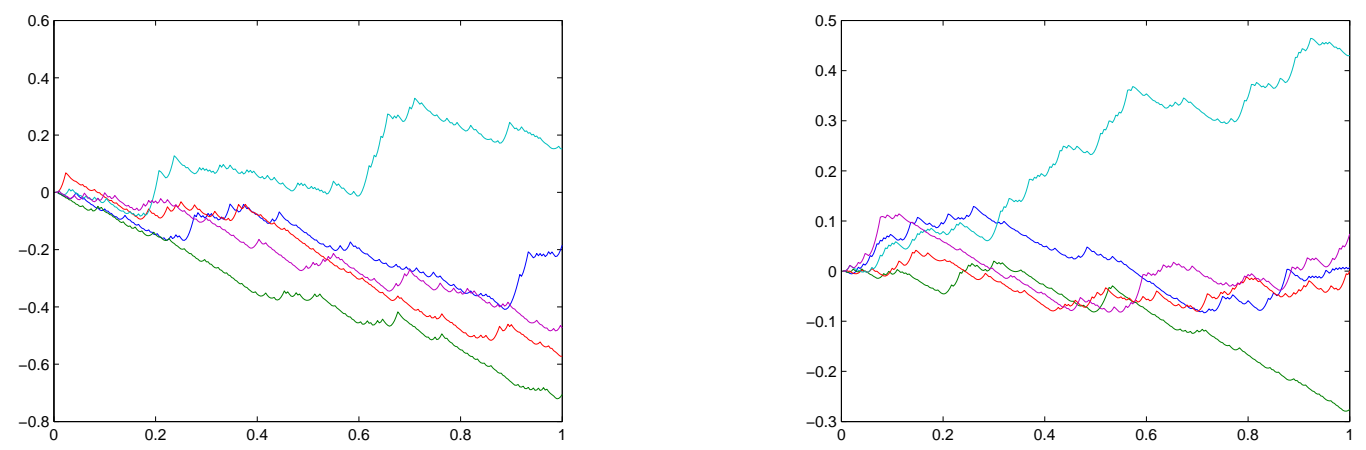

Figure 2: Simulations of the Trajectory for the Rosenblatt process with $H=0.8$ and $H=0.9$.

Acknowledgments: This work was partially supported by the research project Nucleus Millenium P04-069-F "Information and Randomness: Fundamentals and Applications; 
Laboratories in Mathematics of Genome and Stochastic Simulation". The first author was supported partially by the research project Fondecyt Reg. N. 1050843, Chile and Proyecto Anillo ACT-13: "Laboratorio ANESTOC".

\section{References}

[1] P. Abry and V. Pipiras (2006): Wavelet-based synthesis of the Rosenblatt process. Signal Processing, 86(9), pag. 2326 - 2339.

[2] F. Biagini and B. Oksendal (2003): Minimal variance hedging for fractional Brownian motion. Methods Appl. Anal. 10 (3), pag. 347-362.

[3] R. Cont (2006): Long range dependence in financial markets. Preprint, available online http://www.cmap.polytechnique.fr/ rama/papers/FE05.pdf

[4] R. Coviello and F. Russo (2006): Modeling financial assets without semimartingales. Preprint.

[5] F. Delbaen and W. Schachermayer (1994): A general version of the fundamental theorem of asset pricing. Math. Ann., 300(3), pag. 463520.

[6] R.L. Dobrushin and P. Major (1979): Non-central limit theorems for non-linear functionals of Gaussian fields. Z. Wahrscheinlichkeitstheorie verw. Gebiete, 50, pag. 27-52.

[7] P. Guasoni (2006): No arbitrage under transaction costs, with fractional Brownian motion and beyond. Mathematical Finance, 16(3), pag. 569-582.

[8] Y.Z. Hu and P.A. Meyer (1988): Sur les intégrales multiples de Stratonovich. Séminaire de Probabilités XXII, Lecture Notes in Mathematics, pag. 72-81.

[9] Y.Z. Hu and B. Oksendal (2003): Optimal consumption and portfolio in a Black-Scholes market driven by fractional Brownian motion. Infin. Dimens. Anal. Quantum Probab. Relat. Top. 6(4), pag. 519-536.

[10] A. Lo (1991): Long memory in stock market prices. Econometrica, 59, pag. 1279-1313.

[11] B. Mandelbrot (1963): The variation of certain speculative prices. Journal of Business XXXVI, pag. 392-417.

[12] A. Nieminem (2004): Fractional Brownian motion and Martingale differences. Statistics and Probability Letters, 70, pag. 1-10.

[13] I. Nourdin (2005): Schémas d'approximation associés à une équation différentielle dirigée par une fonction höldérienne; cas du mouvement brownien fractionnaire. C.R. Acad. Sci. Paris, Ser. I 340 (8), pag. 611-614. 
[14] I. Nourdin and C.A. Tudor (2006): Some linear fractional stochastic equations. pag. Stochastics 78 (2), 51-65.

[15] D. Nualart (1995): Malliavin Calculus and Related Topics. Springer.

[16] V. Pipiras (2004): Wavelet type expansion of the Rosenblatt process. The Journal of Fourier Analysis and Applications, 10(6), pag. 599-634.

[17] C. Rogers (1997): Arbitrage with fractional Brownian motion. Math. Finance, 7, pag. 95-105.

[18] F. Russo and P. Vallois (1993): Forward backward and symmetric stochastic integration. Prob. Theory Rel. Fields, 97, pag. 403-421.

[19] F. Russo and P. Vallois (2000): Stochastic calculus with respect to a finite quadratic variation process. Stochastics and Stochastics Reports, 70, pag. 1-40.

[20] D. M. Salopek (1998): Tolerance to Arbitrage. Stochastic Proc. Applic., 76(2), pag. 217230.

[21] J.L. Sole and F. Utzet (1990): Stratonovich integral and trace. Stochastics and Stochastics Reports, 29 (2), pag. 203-220.

[22] T. Sottinen (2001): Fractional Brownian m!otion, random walks and binary market models. Finance and Stochastics, 5, pag. 343-355.

[23] M. Taqqu (1975): Weak convergence to the fractional Brownian motion and to the Rosenblatt process. Z. Wahrscheinlichkeitstheorie verw. Gebiete, 31, pag. 287-302.

[24] M. Taqqu (1979): Convergence of integrated processes of arbitrary Hermite rank. Z. Wahrscheinlichkeitstheorie verw. Gebiete, 50, pag. 53-83.

[25] C.A. Tudor (2006): Analysis of the Rosenblatt process. Preprint, SAMOS, Université de Paris 1.

[26] W. Willinger, M. Taqqu and V. Teverovsky (1999): Long range dependence and stock returns. Finance and Stochastics, 3, pag. 1-13.

[27] M. Zaehle (1998): Integration with respect to fractal functions and stochastic calculus. Prob. Theory Rel. Fields, 111, pag. 333-374. 